\title{
The Effect of Teambuilding on Team Development: A Quasi-Experiment within a Swedish State Authority
}

\author{
Christian Jacobsson*, Linnea Nissling, Lisa Skår and Trevor Archer
}

Department of Psychology, University of Gothenburg, Gothenburg, Sweden

\begin{abstract}
The effect of teambuilding on team development was studied with a quasi-experimental design by evaluating consultants' teambuilding interventions in 20 experimental groups within a Swedish state authority. These were compared to 22 control groups that did not receive an intervention. Team development was assessed through pre- and post-test with the "Group Development Questionnaire" (GDQ). Results indicated that experimental groups had significant higher values on scale IV in the GDQ in the post-test compared to control groups. There were no significant differences between experimental and control groups on GDQ scale I-III across time. The results indicate that teambuilding contributes to team development and encourages to further research with regard to "real life" work groups.
\end{abstract}

Keywords: Teambuilding; Team development; Quasi-experiment; Experiment-control group design; Field study

\section{Introduction}

To a great extent, groups may be viewed as the oldest form of social organizations wherein humans have congregated to coordinate experience and resources towards a common goal [1]. From a historical perspective, organization within groups has contributed uniquely to human development and success leading to present-day achievements [2]. Current working life conditions are almost exclusively dependent upon team-based work whereby individuals collaborate in order to carry out ever-increasingly complex assignments [2]. Within group research, several definitions of the group and team concepts are applied. The present treatise, focused upon work groups, maintains the definition of team as: three or more persons working together in order to complete assignments and achieve common goals. Team members are mutually dependent upon each other with complementary roles and tasks. Thus, it is obvious which individuals belong to the group and which do not $[3,4]$.

An overwhelming literature indicates the advantages of team-based work, both for the organization and the individual $[2,5]$. Nevertheless, evidence indicates that it is not sufficient simply to distribute individuals into a group and expect the group to develop into a well-functioning team [2]. In order to develop its full potential a group must acquire the right conditions and necessary support:

"However, the simple existence of a team-based organizing structure is not enough to ensure that positive outcomes will result. Teams must be nurtured, supported, and developed" [2]. The purpose of the present study was to examine if a teambuilding program, led by consultants, contributed to team development among 20 experimental groups compared to 22 control groups in a Swedish state authority.

One of the most common types of intervention regarding group development is "Team-building" [2], the influence of which is analyzed in the present study. Despite the broad application of these interventions, the research concerning efficacy of team-building and which interventions have proven beneficial and why has been limited [2]. Furthermore, the likelihood of generalizing findings from previous research to current occupational conditions has been questioned due to serious limitations pertaining to method and selection $[2,6,7]$. The present study aims to contribute aspects of group-development research that explore how team-building interventions may influence the evolution of groups through the analysis of data derived from a public sector group development project. The project was implemented using an experimental- and control-group design wherein forty-two groups experienced pre- and post-measurements through application of the "Group Development Questionnaire" instrument to determine developmental/maturity levels according to "The Integrated Model of Group Development" [1]. Half of the studied groups were given consultant support for group-development whereas the other half were not given any development support during the project time.

\section{The Integrated Model of Group Development, IMGD}

This study is based on the Integrated Model of Group Development (IMGD) and the linked instrument Group Development Questionnaire (GDQ) [8], as a way of describing and measuring different degrees of team development. The integrated model is an integration of earlier theory and research on team development across time [7,9-12]. The validity of the IMGD and GDQ has been established in a number of studies [8,13-15]. IMGD is a model describing four stages of group development. The stages are (I) dependency and inclusion, (II) counterdependency and fight, (III) trust and structure, and (IV) work and productivity.

The first stage is characterized by team member dependency on the leader, safety concerns, and inclusion issues. The second stage is distinguished by team members having opposing perspectives, counterdependency toward the leader, and tensions in the team. The third stage is distinguished by increased trust and focus on finding better structure and strategies for goal achievement in the team. Finally, the fourth stage is characterized by the intense focus of team members on achieving

*Corresponding author: Jacobsson C, Department of Psychology, University of Gothenburg, 40530 Gothenburg, Sweden, Tel: + +46 31786 1669; E-mail: christian.jacobsson@psy.gu.se

Received: August 14, 2017; Accepted: August 31, 2017; Published: September 07, 2017

Citation: Jacobsson C, Nissling L, Skår L, Archer T (2017) The Effect of Teambuilding on Team Development: A Quasi-Experiment within a Swedish State Authority. Clin Exp Psychol 3: 164. doi: 10.4172/2471-2701.1000164

Copyright: (C) 2017 Jacobsson C, et al. This is an open-access article distributed under the terms of the Creative Commons Attribution License, which permits unrestricted use, distribution, and reproduction in any medium, provided the original author and source are credited. 
the goal(s). Stage IV groups have also established a team climate of openness and cohesion that facilitates effective work. Stage I groups typically spend about $40 \%$ working effectively and Stage IV groups about $80 \%$ of the time. The remaining time is used for maintenance, and dealing with interpersonal issues that arise and the like [15].

Groups characterized by greater development/maturity (i.e., more of stage IV work) are associated with higher levels of goal attainment $[14,16,17]$ and well-being among the group members $[18,19]$. Available data on distribution across stages based on 764 work groups' representative of Swedish working life shows that $29 \%$ of the groups occupy stage I, $21 \%$ in stage II, $30 \%$ in stage III and $20 \%$ in stage IV [20].

\section{Team-Building}

Group-development may be divided into two overall categories: team-building and team-training [21]. Shuffler et al. [21] imply that interventions for team-building and team-training both aim at increasing team efficacy yet focus upon different types of teams and therewith the different requirements of teams. Team-training aspires to the preparation of loose and transient 'patchwork' teams into units that are able to collaborate [21] through the consolidation of groupmembers acquiring co-operative skills such as communicative, which implicate the inclusion of practical training within specific contexts that are specific to the work or task [2]. In contrast to team-training, team-building is described as most effective when a team has a specific problem that hinders the team in its work and thus shows most utility for stable groups composed of the same members over time that have long experience of working together [21]. Team-building is generally less structured with the purpose of of teaching groups basic accomplishments that are important for collaborative enterprise [22]. It is constructed according to the notion that group-members are facilitated by themselves making diagnosis and identification of problems in order to regulate their own behavior [21].

Currently, team-building defines one of the most common interventions for group development [2]. The interventions are developed initially with the aim of allowing groups to evolve and improve their social and interpersonal relations but eventually focus upon several areas of group-development, such as clarification of common goals, achieve results or complete assignments [21]. According to Klein et al. [2] and Shuffer et al. [21], team-building interventions may be sorted into four different categories, each aiming at: goal-clarification, role-clarification, problem-solving and improved interpersonal relations.

In a meta-analysis, Klein et al. [2] examined the effects of teambuilding on cognitive, affective, process- and performance-measures and observed that the intervention induced a moderately positive effect on all the parameters with greatest effect upon affective and process-measures. Taking into account group-size, it was shown that independent of group-size, the interventions were efficacious but with larger group-size were even more efficacious (ibid). A closer study of the different team-building categories indicated that interventions aimed at goal clarification and role-clarification exerted the greatest effects upon outcomes.

Bradley et al. [23] imply that the effect of team-building on group performance is influenced also by the time-frame within which the team is together and the length of time the group is assigned to complete the task. They observed that team-building did not exert any influence for teams that were constructed for only short periods and that were assigned a fictive task during a short period whereas the interventions exerted effects for already-existing teams and newly-constructed teams that worked together on a real task over a more extended period. Within group research, teams assembled for short periods to work on fictive tasks have been applied as a commonplace design which may explain contradictory results that have been obtained for team-building [23]. The present study explores work-groups with assignments within a common area and who worked together over at least three months and are expected to continue to work together during the foreseeable future.

\section{IMGD based team-building interventions}

Studies pertaining to IMGD- and GDQ-based interventions are of particular interest for this article since they are comparable to a great extent with the intervention that is examined in the present study. GDQ-interventions overlap with team-building extensively since they affect goal-clarification and role-clarification, with the inclusion of consideration for the groups' phase of development. Furthermore, the usual interventions affecting communication, feedback, trust and leadership are usually included. Jacobsson and Wramsten W [24] studied teambuilding among teams of high school teachers. From the groups' phase-profile, group-specific focus areas were chosen to allow further development. The focus areas typically consisted of goal-formulation, role-clarification, decision-making processes and construction of functional sub-groups as well as questions regarding leadership. The results, in terms of differences among the four GDQ scales before and after intervention, indicated significant difference on all four scales implying successful group development [24].

\section{Purpose and Problem}

The present study examines the extent to which a consult-training with team-building intervention may influence group development. The experimental groups were assigned to receive a directed teambuilding intervention during the course of a whole day followed by digital 'follow-up' of commitments during a period of six months, and ended by a half-day evaluation. The control groups were not assigned to any intervention but only answering the GDQ at the same point of time as the experimental groups. The problem posed was: does teambuilding intervention influence 'group-maturation' in work groups?

\section{Method}

\section{Participants}

The participants in the present study consisted of 49 work groups within a Swedish state authority that were divided into three areas with 387 workers from various different backgrounds. They were selected from different professions that included for instance veterinary surgeons, legal officers and communicators. The analysis was based upon 42 groups and 297 participants from the original 49 groups, with seven groups excluded. Exclusion criteria pertained to: insufficient criteria for group definition (4 groups), insufficient 'after' measurements (2 groups) and one group was dissolved during the course of the study. Of the groups studied, there were 20 experimental groups and 22 control groups. The groups were composed of all the groups within the governmental administrative organization and assigned to each respective experimental and control condition on a convenience sample basis, what was practically feasible for the organization.

The number of group-members varied between 3 and 16 participant (mean 7.1), with the experimental group consisting of 3-10 (mean 6.4) and the control group between 3 and 16 (mean 7.7). There was a female dominance in the groups, among the experimental groups 
70 percent were female workers and among the control groups 57 percent were female workers. However, according to earlier research gender composition does not seem to account for differences in team development [8]. Prior to the study, the groups had worked together for between 7 and 24 months (mean 12.2) with the experimental groups between 2 and 10 months (mean 7.7) and the control groups between 9 and 24 months (mean 16.6). Table 1 presents an overview of group-member assignment to the experimental and control groups, respectively.

\section{Instrument}

In order to measure groups before and after intervention development, the GDQ SE3 instrument was used, the third Swedish translation of "Group Development Questionnaire" (GDQ) [25], based upon "The Integrated Model of Group Development" [26]. The instrument consists of a questionnaire with 60 statements divided into four scales wherein each scales implies a phase according to IMGD [26]. Each scale comprises 15 statement measuring the presence or absence of typical behavior for each respective phase [26]. The statements in GDQ I measure the degree of energy that the group invests upon issues like 'dependence' and 'inclusion', GDQ II the extent of 'conflict' and 'counter-dependence', GDQ III the extent of 'trust' and 'structure', and GDQ IV the energy extent for 'goal-achievement' and 'work-assignments' [26]. The statements were estimated on a fivedegree Likert scale, whereby " 1 " signified "never fits this group" and " 5 " signified "always fits this group". The total number of points for each respective scale therewith varied between 15 and 75, whereby low scores were associated with low agreement between group and phase and high scores indicated high levels of concordance between group and phase. Table 2 presents examples of the statements provided for each respective scale on the GDQ. In the reliability tests, Cronbach's alpha for GDQ SE3 for Scale I was 0.77, Scale II 0.90, Scale III 0.81, and Scale IV 0.87 [25]. Table 3 presents the norm data for GDQ SE3 which is based upon 764 Swedish groups that were adjudged to be representative for Swedish occupational conditions [19,25].

In order to determine consistency, group means were compared for each respective scale against the norm data for the Swedish population [19]. Consistency was estimated according to the scale whereby group mean was highest in relation to the norm data [25]. Less developed groups expressed high mean levels on scales I and II and low levels on scales III and IV in relation to the norm data whereas groups showing greater development expressed high mean levels on the latter scales and lower levels on the former scales.

\section{Procedure}

Data collection was performed using the GDQ to establish the developmental phase of the groups during pre- and post-interventional measurements. The former were performed during September-toOctober 2015 and the latter during march-aril 2016 with a six-month interval. Each group member completed the GDQ-questionnaire online, individually and anonymously, after which each group-result was calculated on the basis of the individuals' mean-summation on each respective GDQ scale. The questionnaires were administered and assembled by representatives of the consultant company and representatives of the governmental department.

\begin{tabular}{|c|c|c|c|}
\hline & Total-groups & Experiment-groups & Control-groups \\
\hline No. of members, distribution & $3-16$ & $3-10$ & $3-16$ \\
\hline Members, means & 7.1 & 6.4 & 7.7 \\
\hline Age groups, distribution & $3-24$ & $7-10$ & $3-24$ \\
\hline Age groups, means & 12.2 & 7.7 & 16.6 \\
\hline
\end{tabular}

Table 1: Data pertaining to the number of members and functional months for 42 groups.

\begin{tabular}{|c|c|}
\hline GDQ scale & Sample items \\
\hline \multirow{3}{*}{ GDQ 1} & Members tend to go along with whatever the leader suggests. \\
\hline & There is very little conflict expressed in the group. \\
\hline & We haven't discussed our goals very much. \\
\hline \multirow{3}{*}{ GDQ 2} & People seem to have very different views about how things should be done in this group. \\
\hline & Members challenge the leader's ideas. \\
\hline & There is quite a bit of tension in the group at this time. \\
\hline \multirow{3}{*}{ GDQ 3} & The group is spending its time planning how it will get its work done. \\
\hline & We can rely on each other. We work as a team. \\
\hline & The group is able to form subgroups, or subcommittees, to work on specific tasks. \\
\hline \multirow{3}{*}{ GDQ 4} & The group gets, gives, and uses feedback about its effectiveness and productivity. \\
\hline & The group acts on its decisions. \\
\hline & This group encourages high performance and quality work. \\
\hline
\end{tabular}

Table 2: Sample items for GDQ.

\begin{tabular}{|c|c|c|c|c|}
\hline & Scale 1 & Scale 2 & Scale 3 & Scale 4 \\
\hline Max. value & 52.8 & 62.3 & 74 & 72.2 \\
\hline 84 percentile & 43.5 & 43.5 & 59.5 & 61.1 \\
\hline Mean. value & 37.7 & 34.8 & 53.2 & 54.7 \\
\hline 16 percentile & 31.8 & 26.1 & 46.9 & 48.3 \\
\hline Min. value & 20.3 & 16 & 30 & 30 \\
\hline Stand. dev. & 5.8 & 8.6 & 6.3 & 6.3 \\
\hline
\end{tabular}

Table 3: Norms for GDQ SE3 based on 764 Swedish work groups. 


\section{Experimental design}

An experimental and control group design whereby 20 experimental groups underwent directed consultant-training for group development was applied whereas 22 control groups were not recipient of this intervention. The consultant-training consisted of a whole-day together with the groups wherein the groups were provided initially with a theoretical framework of "The Integrated Model of Group Development" [1] as well as a presentation of the groups' individual GDQ-profile from the pre-interventional measurement. Later, the teams worked upon the five factors incorporated in the consult-firm concept: goal-precision, advantageous structures, open interactions, positive development cultures and high commitment. From the groups' phase profiles (GDQ) together with discussions emanating from the five factors essentially, the groups were assigned a general exercise based on three themes: (i) the goal(s), whereby different issues were posited, e.g. "Which goals do we want to achieve together, within six months, or one year?". The goal could consist of both developmental or deliverance goals, for example including "How may we become a phase IV team?" and "How may we achieve a better cooperation?"; (ii) the structure, with the posited issue "How may we work even more effectively together in order to achieve our goals?". The structure discussion included issues pertaining to how the groups may evolve more productive meetings, functional follow-up processer and continual self-monitoring; (iii) the game-rules, positing issues such as "How may we behave in relation to each other?". The game-rules under discussion included for instance feedback and professionalism with the purpose of agreement within the group regarding how each person related to the others in the group. The groups' discussions around these themes led to each group developing its own 'action-plans' for continued collaboration. During six months, follow-up on the 'action-plans' was carried out in the groups using a digital tool delivered by the consultants, whereby the groups were allowed to evaluate and provide feedback regularly concerning how well they experienced the survival of the existing 'action-plans'. The consulttraining episode was terminated with a half-day follow-up wherein the groups were given a reinstatement of assignments combined with the presentation of the post-interventional results from GDQ.

\section{Statistical analysis}

In order to examine the extent to which the group-development interventions influenced group maturation, differences in the means of the GDQ scales I-IV between the experimental and control groups were analyzed at the pre- and post-interventional measurements using two-way ANOVA with repeated measures (mixed design) examine for interaction effects. To test for "simple effects" of the experimental and control groups, respectively, between the pre- and post-interventional measurements, the data were 'split' after which one-way ANOVA was performed upon those scales a significant interaction effect had been obtained.

\section{Results}

Consult-training with team-building interventions was shown to have induced an influence upon the development of the groups under study, i.e., whether not there was a difference in group development on the post-intervention tests between the experimental and control groups. Table 4 presents mean values the experimental and control groups, respectively, on each of the GDQ scales during the pre- and post-interventional measurements. In comparison with the Swedish norm data (Table 3), it was found that the pre-intervention measures of the experimental group were lower on Scales I and II but higher on scales III and IV. It is indicated that the experimental group expressed

\begin{tabular}{|c|c|c|c|c|}
\hline Scale: GDQ & GDQ I & GDQ II & GDQ III & GDQ IV \\
\hline Control pre-measure* $^{*}$ & 37.7 & 34.9 & 52.5 & 53.7 \\
\hline Control post-measure\# $^{*} 37.2$ & 34.9 & 52.7 & 53.9 \\
\hline Experiment pre-measure* $^{*}$ & 33.1 & 30.0 & 54.7 & 55.9 \\
\hline Experiment post-measure\# $^{*}$ & 32.2 & 30.0 & 56.3 & 58.4 \\
\hline
\end{tabular}

Note: *pre-intervention; \#post-intervention

Table 4: Means for Scales I-VI for experimental and control groups at pre- and post-intervention.

greater development that the norm data for Swedish occupational groups.

In order to examine whether or not there occurred a difference between the experimental and control group on the pre- and postinterventional measurements, a two-way independent ANOVA with repeated measures (mixed design), for scales I-IV of the GDQ, respectively, was performed. There was a significant interaction effect for the GDQ Scale IV F $(1,40)=4.25, p<0.05$, partial $\eta^{2}=0.10$; but not for GDQ Scale I $F(1,40)=1.23, p>0.05$, partial $\eta^{2}=0.03$; GDQ Scale II $\mathrm{F}(1,40)=0.00, \mathrm{p}>0.05$, partial $\eta^{2}=0.00$ or GDQ Scale III $\mathrm{F}(1,40)=1.38$, $p>0.05$, partial $\eta^{2}=0.03$. Following the significant interaction effect for GDQ Scale IV, the presence of "simple effects" for the experimental and control groups was tested for separately to ascertain whether or not the group-development interventions had induced alterations in the desired direction while there was an absence of these alterations in the control groups. It was indicated that the mean value of the experimental group on GDQ Scale IV was significantly higher post-intervention: $\mathrm{F}(1,19)=7.50, \mathrm{p}<0.05$, partial $\eta 2=0.28$, indicating a medium strong effect size [35] whereas for the control groups there were no significant difference: $\mathrm{F}(1,21)=0.04, \mathrm{p}>0.05$, partial $\eta 2=0.00$.

\section{Discussion}

The present study examined whether or not consult-training with team-building interventions may influence group development among work groups within a Swedish state authority. The results aimed to provide an answer to the problem: does team-building intervention influence 'group-maturation' in work groups? It was indicated that the experimental group increased significantly its' scoring on the GDQ Scale IV scoring from the pre- to the post-interventional phase implying that the team-building interventions influenced it according to Wheelan [1], whereby the degree of energy a group invests upon its behavior characterizes an effective team. This notion posits also that a group that has matured to a greater level concurrently reduces its mean values on GDQ Scales I and II [13], although significance for this result was not obtained, implying further efforts for development were necessary for the groups involved. Thus, progress amongst issues involving dependency and inclusion, conflicts, counter-dependence, and work-structure was lacking. In view of the marginally higher development of the experimental group compared to the Swedish norm data on scale IV that may have influenced the outcome could imply that the groups had advanced somewhat and this essentially interfered with development on the other scales. The mean values on scale I and II were already low in the pre-measures.

How may this result be understood from the context of previous findings pertaining to team-building effects? Earlier findings are contradictory with regard to if, how and why interventions function $[2,27]$. They have studied the outcome of team-building efficacy to the exclusions of experimental and control group designs with the majority of group involving students and training groups which questions the generalizability of those findings [2], whereas the present study fulfils 
the empirical necessities regarding design, population and outcome. Nevertheless, meta-analyses [2,27] underline the significance of this study for cognitive, affective, process- and personal-measures to describe what has been construed to be an 'effective team' [1]. Several studies have demonstrated the association between team-development and performance $[1,15-17,28,29]$, and also between team-development and affective indications such as work-satisfaction, emotional exhaustion and sick leave $[18,19,30]$. Effective teams are characterized by behaviors such as information exchange, functional leadership alternation and metacognition centered around the groups' work, problems and solutions, that include process- and cognitive-indices as outcomes [21].

It is important to examine the extent to which the consultant company's interventions relate to team-building interventions studied earlier. The present intervention was derived from the notion of the consultants teambuilding concept with comparative similarities to factors, role clarification and goal setting [2]; to some extent the intervntions are also comparable to interpersonal relationship interventions although the latter remain less effective as the Klein et al. [2] meta-analysis indicated. GDQ-based interventions influenced group-development in both the Buzaglo and Wheelans [31] and Jacobsson and Wramsten Wilmar [24] studies wherein interventions were adapted to the groups' phase and members' discussion around their common development areas. Buzaglo and Wheelan [31] imply that psycho-education about development theory and effective teambehaviors rather than individual training is essential for effective groupdevelopment, which comprises a part of the consults intervention whereby IMGD theory is presented. GDQ-based interventions usually also involve communicative behavior and feedback [24]. Varieties of communicative behavior and their correlation with work -satisfaction, productivity and organizational success have been investigated by Kauffeld and Lehmann-Willenbrock [32]. They observed that groups that during meetings: (i) identified problems on the basis of causation, consequences and possible solutions, (ii) kept to a schedule, time-frame, clarifications of their own and others statements and summarized conclusions and decisions, and (iii) took an interest in change, maintained personal responsibility and action-decisions, had greater work satisfaction, productivity and organizational success. These 'communicative' behaviors align with some aspects of the consultants concept in the present study. For instance the concept of favored structures wherein the weight of effective meeting structure and decision- and information-avenues are imperative, where through heightened engagement group members are challenged to take personal control provide instances of team-building interventions reported in earlier research $[2,27,32]$.

In summary, team-building interventions may exert positive influences upon group-development, yet the question of how this is achieved remains. In order to develop into effective teams, the organizational and contextual conditions ought to be fulfilled $[4,22]$. Accordingly, it seems that the conditions surrounding the state authority may contributed to the successful group-development measured postintervention due to both working together as effective team and lower estimations on earlier scales of GDQ. It is noticeable that one quarter of the experimental groups, belonged to a department that differed from the other departments with regard to leadership due to missing their department-leader [33], which could have reduced the interventional effects under a provisional leader. Nytro et al. [34] imply that both motivation and trust in the intervention affect outcome in addition to intervention syntax. Furthermore, Wheelan [4] indicates that an essential ingredient is the precis definition of goals within assignment.
All of which supports the notion of the 'provisional leader' effect, emphasizing the 'leadership-aspect'. In the present study, differences emerged between the experimental and control groups both for the periods when they worked together and in the distribution of members which may have 'opened-the-door' to conditions for differential groupdevelopment conditions. The relevance of these factors for the teambuilding interventions with regard to experimental-control group differences may be discussed. The latter worked together, on average, twice as long as the former with a larger number of group-members. The natural group-development tends to be greatest initially during the first year of cooperation and then tapering off which seems the case with the experimental groups. Regarding group size, Klein et al. [2] indicate that efficacy and decision-propensity are affected by group size: larger groups indulge in more discussion leading to delayed/questionable decisionmaking. The optimum number of group-members is a condition for a well-function team [3]. Taking into account the potential effects of age and group-member size, it is possible the control groups had worse conditions for group-development which implies that the experimental groups may not necessarily have progressed to higher extent due to the intervention solely.

\section{Limitations}

Due to administrative constraints of an ongoing project, the design of group-development interventions and data-collection lacked sufficient control by the authors not least since group-assignment followed a 'least-resistance' pathway which resulted in a lack of randomization and sufficient matching from aspects of membership size, amount of time together, or leadership, all of which may have influenced the result. Group differences may be negligible for the project at large, due to the use of a 'cross-over' design in a later stage of the project than reported here, resulting in approximately 40 experimental and 40 control groups. Group-size differences, however, remain an issue. Team-building generally produces greater effects with larger groups [2] which would suggest larger effects in the planned 'cross-over' design study. A further limitation pertains to the outcome measures, GDQ, presented as questionnaire allowing it to be colored by group-members' subjective reporting [2]. Nevertheless, GDQ offers a well-established instrument with high reliability and validity [8] with several studies presenting high concordance between GDQ estimations and objective performance measures [1,15-17,29]. The study gives a 'half-way' mark of the complete project with relatively limited sample which also may have lack of sufficient statistical power.

\section{Conclusions and Recommendations}

The present study contributes to research on the effectiveness of team-building by demonstrating that there was a positive effect of group-development expressed by the higher level of efficacy shown by the experimental group post-intervention. Nevertheless, this result ought to be interpreted with caution since only a significant effect was observed on GDQ Scale IV but not on the other scales, I-III. Due to methodological and conditional constraints, it cannot be precluded that factors other than intervention affected the result (see above). However, as one of the few experimental-control group designs upon pre-existing work groups, there is some potential for generalization to Swedish state authority conditions. These present results are relevant to workers and employers undertaking investment decisions pertaining to group-development initiatives. Implication for policy is that, although the results in the study only provide modest indications that team development is an effective investment to streamline the work of government agencies, there are good reasons to continue doing this 
Citation: Jacobsson C, Nissling L, Skår L, Archer T (2017) The Effect of Teambuilding on Team Development: A Quasi-Experiment within a Swedish State Authority. Clin Exp Psychol 3: 164. doi: 10.4172/2471-2701.1000164

kind of experiments. The results encourage a continued exploration.

\section{References}

1. Wheelan SA (2005) Group processes: a developmental perspective. Allyn and Bacon, Boston.

2. Klein C, DiazGranados D, Salas E, Le H, Burke CS, et al. (2009) Does team building work? Small Group Research 40: 181-222.

3. Hackman JR (2012) From causes to conditions in group research.J Organizational Behav 33: 428-444.

4. Wheelan SA (2013) Att skapa effektiva team - En handledning för ledare och medlemmar. (2:a upplagan) [Creating Effective Teams]. Studentlitteratur, Lund.

5. Levi D (2010) Group dynamics for teams,SAGE, London.

6. Chidambaram L, Bostrom RP (1997) Group development (I): A review and synthesis of development models. Group Decision and Negotiation 6: 159-187.

7. Tuckman BW, Jensen MAC (1977) Stages in small group development revisited. Group and Organization Studies 2: 419-427.

8. Wheelan SA (1996) Effects of gender composition and group status differences on member perceptions of group developmental patterns, effectiveness, and productivity. Sex Roles 34: 665-686.

9. Bales RF (1950) Interaction process analysis. Addison-Wesley, Cambridge MA.

10. Bales RF, Strodtbeck FL (1951) Phases in group problem-solving. J Abnorm Soc Psychol 46: 485-495

11. Bennis WG, Shepard HA (1956) A theory of group development. Human Relations 9: 415-437.

12. Bion WR (1961) Experiences in groups. Tavistock, London.

13. Wheelan S, Burchill C, Tilin F (2003) The link between teamwork and patients outcomes in intensive care units. Am J Crit Care 12: 527-534.

14. Wheelan SA, Davidson B, Tilin F (2003) Group development across time: Reality or illusion? Small Group Research 34: 223-245.

15. Wheelan SA, Williams T (2003) Mapping dynamic interaction patterns in work groups. Small Group Research 34: 443-467.

16. Wheelan S, Kesselring J (2005) The link between faculty group development and the performance of elementary students on standardized tests. The Journal of Educational Research 98: 323-330

17. Wheelan S, Murphy D, Tsumura E, Fried Kline S (1998) Member perceptions of internal group dynamics and productivity. Small Group Research 29: 371-393.

18. Jacobsson C, Rydbo N, Börresen JE (2014) The links between group development and health in manufacturing industry. Small Group Research 45: 400-415.

19. Jacobsson C, Åkerlund M, Graci E, Cedstrand E, Archer T (2016) Teacher team effectiveness and teachers well-being. Clin Exp Psychol 2:130.
20. Wheelan SA, Jacobsson C (2014) A descriptive study of work groups in the Swedish and U.S. Economy. Paper presented at the the 9th GRASP conference, Linköping University, Linköping.

21. Shuffler ML, DiazGranados D, Salas E (2011) There's a science for that: Team development interventions in organizations. Curr Dir Psychol Sci 20: 365-372.

22. Forsyth DR (2013) Group dynamics, Wadsworth, Belmont, CA.

23. Bradley J, White BJ, Mennecke BE (2003) Teams and tasks. A tempora framework for the effects of interpersonal interventions on team performance. Small Group Research 34: 353-387.

24. Jacobsson C, Wramsten WM (2009) Increasing teacher team effectiveness by evidence based consulting. Paper Presenterat på 14th European Congress of Work and Organizational Psychology (EAWOP). Santiago Compostela, Spain.

25. Jacobsson C, Persson O (2011) Group development, what's the speedlimit? Two cases of student groups. In C. Jacobsson, \& M. R. Ricciardi (Eds), The individual and the group - Future challenges. Proceedings from the 7th GRASP conference. University of Gothenburg, Gothenburg, Sweden.

26. Wheelan SA, Hochberger JM (1996) Validation studies of the group development questionnaire. Small Group Research 27: 143-170

27. Salas E, Rozell D, Mullen B, Driskell JE (1999) The effect of team building on performance: An integration. Small Group Research 30: 309-329.

28. Wheelan S, Furbur S (2006) Facilitating team development: Communication and productivity. In L. Frey, (Ed.). Facilitating group communication in context: Innovations and Applications with natural groups, (s. 155-176). SAGE Thousand Oaks, CA

29. Wheelan S, Tilin F (1999) The relationship between faculty group effectiveness and school productivity. Small Group Research 30: 59-81

30. Svedlund S (2012) The relationship between groups developmental level and perceived stress, exhaustion and work satisfaction. Unpuplished Master Thesis Psykologprogrammet, Psykologiska institutionen, Göteborgs Universitet Göteborg, Sweden.

31. Buzaglo G, Wheelan S (1999) Facilitating work team effectiveness: Case studies from Central America. Small Group Research 30: 108-129.

32. Kauffeld S, Lehmann-Willenbrock N (2012) Meetings matter: Effects of team meetings on team and organizational success. Small Group Research 43: 130 158.

33. Wigenius M (2016) Personal communication.

34. Nytrø K, Saksvik PØ, Mikkelsen A, Bohle P, Quinlan M (2000) An appraisal of key factors in the implementation of occupational stress interventions. Work and Stress 14: 213-225.

35. Cohen J (1992) A power primer. Psychol Bull 112: 155-159.

36. Eklöf L (2013) A comparison of team development in 54 teams with stabile or changes team-structure. Unpublished Master Thesis. Psykologprogrammet, Psykologiska Institutionen, Göteborgs Universitet, Göteborg, Sweden. 